\title{
A Molecularly Imprinted Polymer Based Biosensor for Electrochemical Impedance Spectroscopic Analysis
}

\author{
Feride Şermin Utku1 ${ }^{1}$, Ozan Enver Özdemir ${ }^{1,2}$, Melahat Sevgül Bakay',3 \\ 'Department of Biomedical Engineering, Yeditepe University School of Engineering, İstanbul, Turkey \\ ${ }^{2}$ Department of Metallurgical and Materials Engineering, İstanbul Technical University School of Engineering, İstanbul, Turkey \\ 3Department of Electrical and Electronic Engineering, İstanbul Technical University School of Engineering, İstanbul, Turkey
}

Cite this article as: F. Ş. Utku, O. E. Özdemir, M. S. Bakay. "A Molecularly Imprinted Polymer Based Biosensor for Electrochemical Impedance Spectroscopic Analysis". Electrica, vol. 18, no. 1, pp. 39-44, 2018.

\begin{abstract}
A molecularly imprinted polymer (MIP)-based impedimetric biosensor was developed for the electrochemical analysis of low-weight biological molecules. Synthetic polymeric matrices with specific and selective recognition sites, which are complementary to the shapes and sizes of the functional groups of analytes, can be prepared using the molecular imprinting method. In this study, a small molecule, tris(hydroxymethyl)aminomethane (TRIS), was used to coat a graphite pencil tip with a TRIS-containing polyacrylamide gel to fabricate a working electrode. The electrode modification and performance were evaluated using cyclic voltammetry and electrochemical impedance spectroscopy. The electrochemical properties of the modified electrodes were observed using an electrochemical cell comprising a Ag/AgCl reference electrode, a Pt wire as the counter electrode, and a pencil graphite tip as the working electrode using a redox-phosphate buffer solution with different concentrations of TRIS and Ethylenediaminetetraacetic acid (EDTA). The I-V and impedance performance of the chemically modified graphite pencil-tip electrodes exhibited decreased conductance and increased impedance correlating with the increase in TRIS concentration. Thus, MIP-based small-molecule biosensor prototypes can be promising economical replacements over other expensive sensors.
\end{abstract}

Keywords: Molecularly Imprinted Polymer, Cyclic Voltammetry, Electrochemical Impedance Spectroscopy, tris(hydroxymethyl)aminomethane, Pencil Graphite Electrode

Address for Correspondence:

Feride Şermin Utku

E-mail:

sermin.utku@yeditepe.edu.tr

Received: 23.08 .2017

Accepted: 23.11 .2017

(c) Copyright 2018 by Electrica

Available online at

http://dergipark.gov.tr/iujeee

DOI: 10.5152/iujeee.2018.1807

\section{Introduction}

Biosensor, a device used in the detection of an analyte, combines a biological/chemical sensor component with a physicochemical transducer. The sensor and the transducer elements recognize and detect the analyte qualitatively and/or quantitatively [1-3]. The biological sensor element may be in the form of tissue, microorganism, organelle, receptor, enzyme, antibody, nucleic acid, molecule, etc., which may be attached to the metal, polymer or glass surface of the electrode through chemical and physical means.

A biological sensor element may be relatively short-lived and with complications in handling; therefore, they may be replaced with artificial elements that are components of a receptor-based sensing system. Molecular Imprinting Technology (MIT) is a method that aims to overcome these complications by producing selectively specific artificial receptors. It utilizes molecular imprinting polymer (MIP), formed as a dependable molecular recognition element with room temperature stability that mimicks natural recognition elements, such as antibodies and receptors. MIT is used in the detection, separation and purification of biological and chemical molecules, such as amino acids and proteins, nucleotids, toxins, drugs, etc. A 3D polymeric network is formed between the analyte and monomer through functional hydrogen bonds, dipole-dipole and ionic interactions. After polymerization, upon removal of the analyte, specific recognition sites that are in the shape, size and chemical structure of the analyte are formed in the polymer [4-11].

There are examples of MIP based sensors equipped more commonly with SPRS and QCM based transducers [4]. However, they have not been experimented in the detection of small 
molecules, such as metabolites formed during the various stages of cellular development using electrochemical transducers. Hydroxymethylaminomethane (TRIS), has been chosen as a small molecule used in established basimetric standard and buffer solutions, commonly used in biochemistry and molecular biology processes, such as protein and nucleic acid extraction and purification [12]. As the basic small molecule, TRIS has been used to produce the MIP electrode coated with TRIS containing polyacrylamide gel (PAGE) to produce TRIS receptor sites.

In this study, a MIP based sensor for TRIS made of pencil graphite electrode (PGE) was combined with electrochemical transduction techniques, i.e. cyclic voltammetry (CV) and electrochemical impedance spectroscopy (EIS) [13]. Upon binding of the analyte to the receptor sites on the gel surface, changes in I-V properties and impedance of the MIP modified PGE were expected to be detected using CV and EIS.

\section{Materials and Experimental Method}

\section{Materials}

Merck analytic quality chemicals were used in this study. For the electrochemical analysis, $20 \mathrm{mM} \mathrm{K}_{3}\left[\mathrm{Fe}(\mathrm{CN})_{6}\right]$ redox pair in phosphate buffer solution was used (redoks-PBS, $137 \mathrm{mM}$ $\mathrm{NaCl}, 2.7 \mathrm{mM} \mathrm{KCl}, 10 \mathrm{mM} \mathrm{Na}_{2} \mathrm{HPO}_{4}, 1.8 \mathrm{mM} \mathrm{KH}_{2} \mathrm{PO}_{4}, \mathrm{pH} 7.4$ ) [14$16]$.

Three types of PGEs were prepared: (a) control electrodes (PGE), (b) positive control electrodes (PAGE-PGE), and (c) experimental electrodes for TRIS analysis (TRIS-PAGE-PGE). All electrodes were initially anodized in acetic buffer solution (ABS, 0.1 $\mathrm{N}$ acetic acid, $0.1 \mathrm{~N}$ sodium acetate, $20 \mathrm{mM} \mathrm{NaCl}, \mathrm{pH} 4.8$ ) for 60 seconds at $+1.4 \mathrm{~V}[15]$.

Five PGEs were used as control electrodes without further processing. PAGE-PGEs were prepared by coating PGEs with $12 \%$ polyacrylamide gel. TRIS-PAGE-PGEs were prepared by coating PGEs with $12 \%$ polyacrylamide gel containing $10 \mathrm{M}$ TRIS.

\section{Instruments}

Anodization and electrochemical analysis were conducted using a potentiostat (ZIVE SP2 Potentiostat/Galvonostat, Referans 600 , Korea) equipped with ZMAN EIS data analysis program. The three-electrode cell consisted of $\mathrm{Ag} / \mathrm{AgCl}$ as the reference electrode, platinum wire as the counter electrode, and PGE as the working electrode. The morphology of the coating on working electrodes was characterized by Scanning Electron Microscope (SEM) (Carl ZEISS EVO40).

\section{Electrochemical Measurement Method}

PGEs, PAGE-PGEs and TRIS-PAGE-PGEs were characterized electrochemically as stated below. Electrochemical measurements were conducted by immersing the working electrodes in solution at a depth of $2.5 \mathrm{~cm}$ and at a distance of $5 \mathrm{~cm}$ from the counter electrode.
Electrode modification was characterized using $\mathrm{K}_{3}\left[\mathrm{Fe}(\mathrm{CN})_{6}\right]$, a commonly used redox couple former $\left[\mathrm{Fe}(\mathrm{CN})_{6}\right]^{4-} /\left[\mathrm{Fe}(\mathrm{CN})_{6}\right]^{3-}$ with well-defined electron transfer kinetics. Cyclic voltammetry was used at an applied potential varying between -500 and 500 $\mathrm{mV}$ relative to $\mathrm{V}_{\text {ref }}$ (step size: $20 \mathrm{mV}$, scan rate: $50 \mathrm{mV} / \mathrm{s}$. I-V cycles were generated by swaying the potential initially anodically, then cathodically. In the forward scan, $\mathrm{Fe}(\mathrm{CN})_{6}^{-4}$, is oxidized, generating an oxidative peak, followed by a reductive peak in the reverse scan [16].

$\begin{array}{ll}\mathrm{Fe}(\mathrm{CN})_{6}^{-4} & \rightarrow \mathrm{Fe}(\mathrm{CN})_{6}^{-3}+\mathrm{e}^{-} \\ \mathrm{Fe}(\mathrm{CN})_{6}^{-3}+\mathrm{e}^{-} & \rightarrow \mathrm{Fe}(\mathrm{CN})_{6}^{-4}\end{array}$

The open cell potential (OCP) was measured in redoks-PBS solution till stabilization. The electrochemical impedance studies were conducted using AC voltage at an amplitude of $+10 \mathrm{mV}$ relative to OCP, between a frequency range of 10,000 and $0.01 \mathrm{~Hz}$.

The CV and EIS response of the TRIS-PAGE-PGEs to the analyte was examined by introduction of incremental amounts of $0.25 \mathrm{M}$ TRIS to determine electrode response to TRIS (400, 600,800 , and $1000 \mu \mathrm{l}$, at a final concentration of 1.0, 1.5, 2.0 and $2.5 \mathrm{mM}$ respectively). To confirm selectivity and specificity of the electrode to TRIS, incremental amounts of $0.25 \mathrm{M}$ Ethylenediamine tetraacetic acid (EDTA) was used (400, 800, and $1000 \mu \mathrm{l}$, at a final concentration of $1.0,2.0$ and $2.5 \mathrm{mM}$ respectively).

\section{Results}

In this study, PGEs were modified to produce PAGE-PGEs and TRIS-PAGE-PGEs. Binding of TRIS to the TRIS-PAGE-PGEs was demonstrated using electrochemical methods as indicated with an increase in impedance. The selective specificity of the TRIS electrode was further confirmed by experiments involving EDTA, indicating that EDTA not bound to the surface increased solution conductivity and reduced impedance.

PGEs were anodized in ABS at $+1.4 \mathrm{~V}$ in order to limit the conductivity of PGEs [17]. In order to standardize the electrical conductivity of the electrodes, the initial $30 \mathrm{sec}$. anodization length (Figure 1) was extended to $60 \mathrm{sec}$. The PGEs were chosen among the low conducting anodized electrodes. Post 60 sec. oxidation, PGE conductivity was sufficiently decreased to values ranging between $42-142 \mu \mathrm{A}$.

The electrochemical properties of PGEs (C1 and E1) and PAGE-PGEs (C2 and E2) electrodes were determined in redoks-PBS solution using CV (Figure 2) and EIS (Figure 3).

The cell potential, the current amplitude and peak potential of PAGE-PGEs decreased with PAGE coating (Figure 2 and 4, C1-C2), while impedance was increased as expected (Figure 3, E1-E2). The I-V behavior of TRIS-PAGE-PGEs (Figure 2 and 4, C3-C6) displayed a decrease in current amplitude and a slight and gradual increase in peak potential and electrochemical cell potential; while in the EIS spectrum an increase in impedance, 


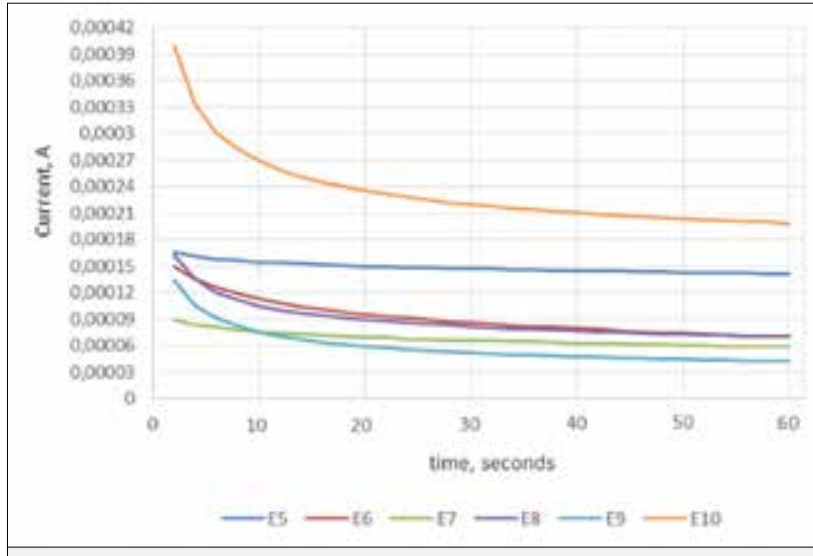

Figure 1. Current-time curve for PGEs anodized in $A B S$ at $+1.4 \mathrm{~V}$

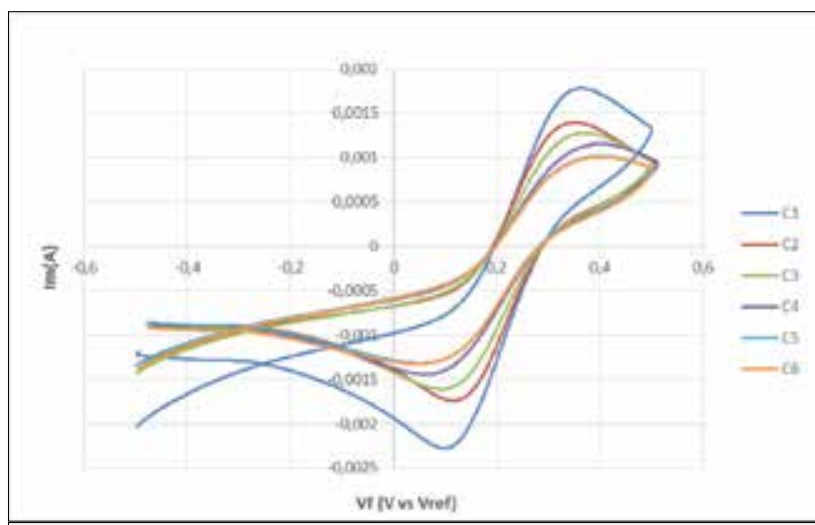

Figure 2. $C V$ graphics in redoks-PBS solution. $C 1$; PGE, $C 2$; PAGEPGE, C3-C6: TRIS-PAGE-PGE in TRIS-redoks-PBS solution; C3; $1 \mathrm{mM}$ TRIS, C4; 1.5 mM TRIS, C5; 2 mM TRIS, C6; 2.5 mM TRIS

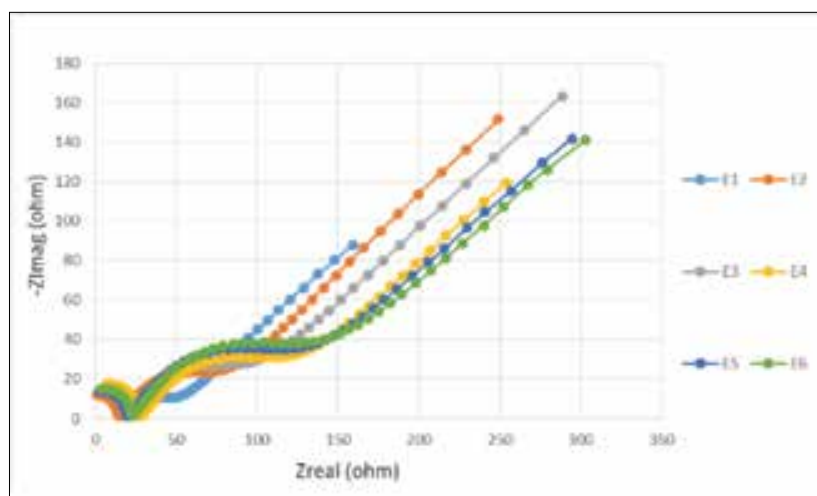

Figure 3. EIS graphics in redoks-PBS solution. E1: PGE, E2: PAGEPGE, E3-E6: TRIS-PAGE-PGE in TRIS-redoks-PBS solution; E3: $1 \mathrm{mM}$ TRIS, E4: 1.5 mM TRIS, E5: 2 mM TRIS, E6: 2.5 mM TRIS

correlating with the incremental increase in TRIS concentration was observed (Figure 3 and 4d, E3-E6).

The incremental increase in the EDTA concentration in redoks-PBS solution did not display a significant effect on the I-V behavior of TRIS-PAGE-PGEs (Figure 5). The EIS spectrum of TRIS-PAGE-PGEs exposed to an increase in EDTA concentration

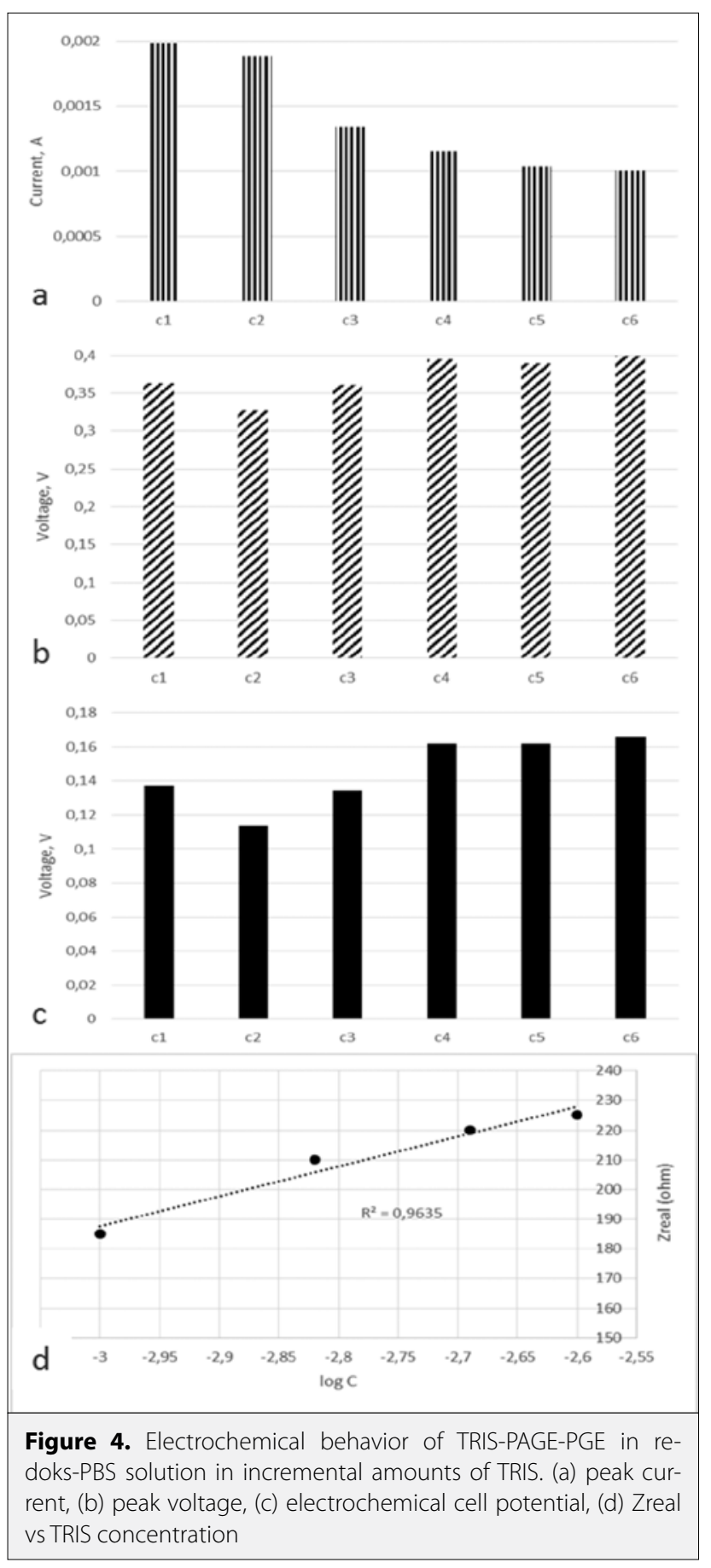

displayed a reduction in impedance (from 400 to $230 \mathrm{Ohms}$ for Zreal and from 225 to 120 Ohms for -Zlmag) with an apparent increase in solution conductivity as observed in the decrease in the radius of the semi-circular portion of the curve (Figure 6).

The morphology of the electrodes displaying the uncoated PGE surface (Figure 7a), the PAGE layer on PAGE-PGE surface (Figure 7b), and the TRIS-PAGE-PGE surface after electrochemical characterization in TRIS-redoks-PBS solution (Figure 7c) was characterized using SEM imaging. 


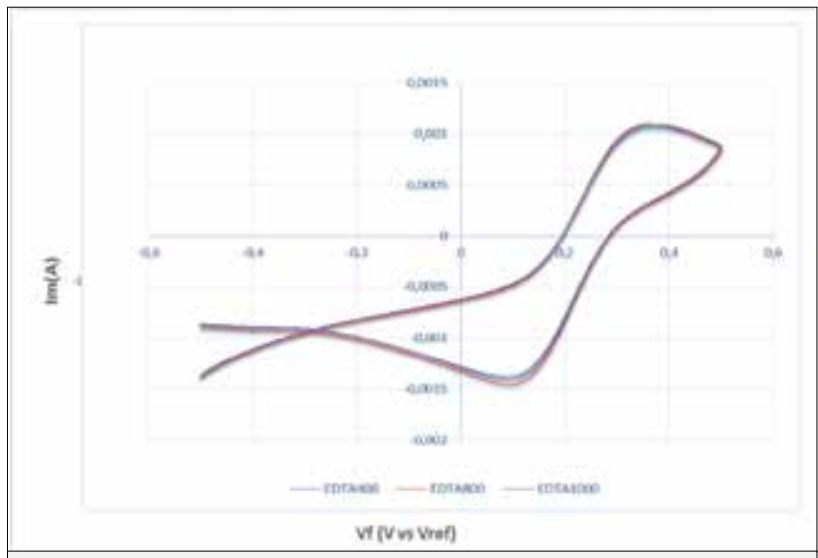

Figure 5. CV graphics for TRIS-PAGE-PGE in redoks-PBS solution in incremental amounts of EDTA in redoks-PBS solution. EDTA400/800/1000 with 1/2/2.5 mM EDTA

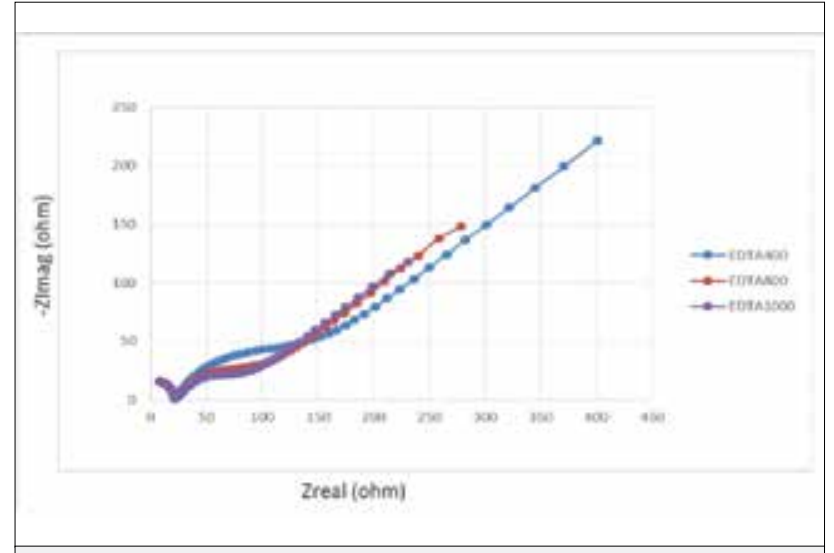

Figure 6. EIS graphics for TRIS-PAGE-PGE in redoks-PBS solution in incremental amounts of EDTA in redoks-PBS solution. EDTA400/800/1000 with 1/2/2.5 mM EDTA

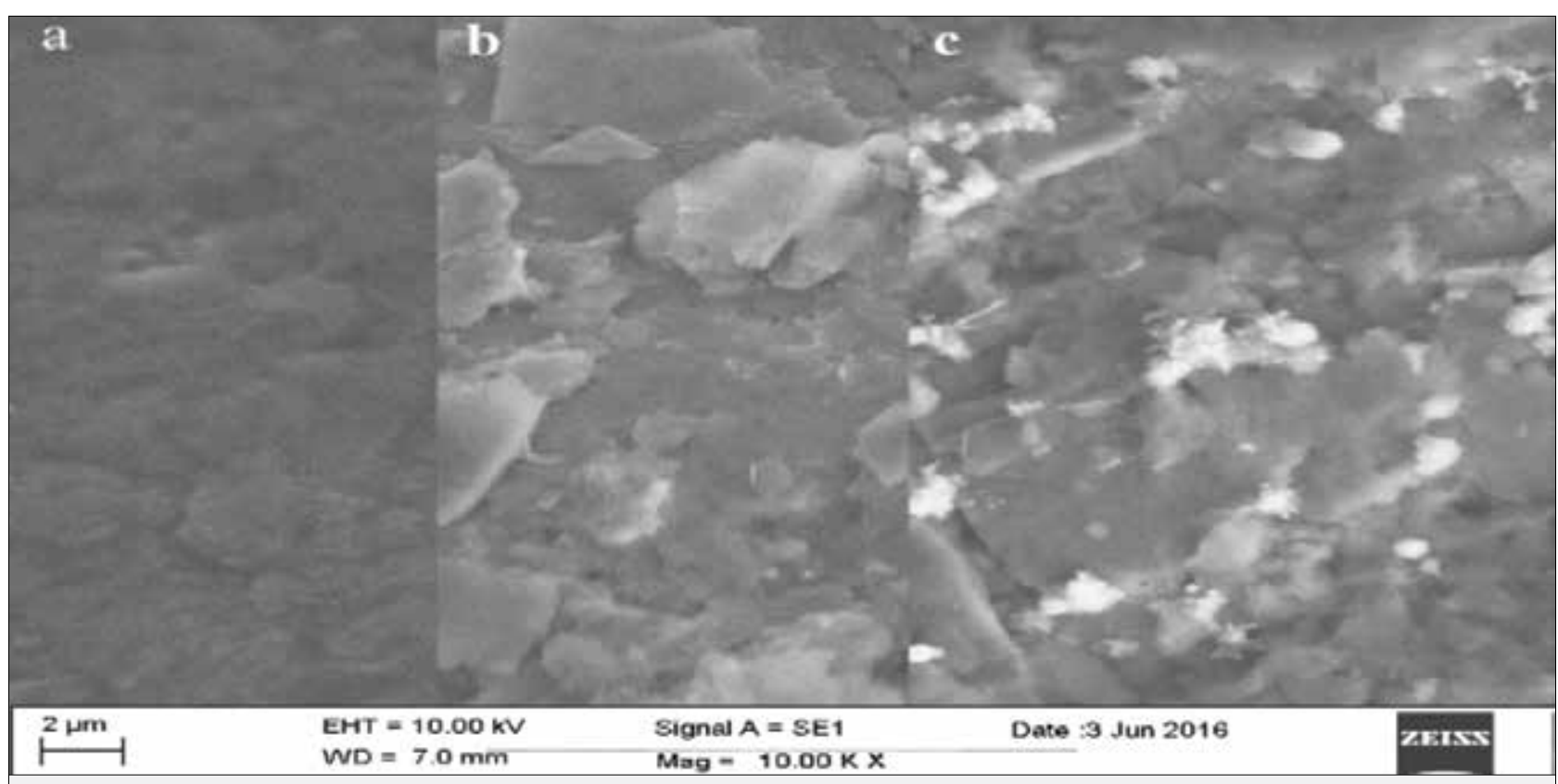

Figure 7. SEM images of (a) plain PGE surface, 10kX; (b) PAGE-PGE coated surface, 10kX; (c) TRIS-PAGE-PGE coated surface with TRIS crystals, $10 k X$

\section{Discussion}

In this study, a prototypic small molecule detecting biosensor, selectively specific to TRIS was produced using MIP-coated PGE as the sensing element and a potentiostat as the transducer in the conductance of CV and EIS analysis.

Graphite pencil leads, modified as PGE working electrodes are composite materials containing graphite ( $65 \%)$, clay ( $30 \%)$, and a binder (wax, resins, or high polymer) [18]. PGEs with high chemical and mechanical stability can be used in a wide range of working potentials [18]. PGEs are cheap, easily modified and convenient to use in quantification of a variety of analytes from a wide range of samples. With an active electrode surface area of approximately $0.255 \mathrm{~cm}^{2}$, they can be used to analyse low concentrations of small volume samples without any preconcentration/deposition step [18].

PGEs are highly durable and stable, yielding reproducible signals and well-defined voltammetric peaks with good sensitivity and reproducibility. Background currents generated by clays and polymers contained in PGEs are much lower than those given by the conventional solid electrodes, such as glassy carbon, gold, and platinum; while displaying electron transfer rates similar to those of glassy carbon electrodes [18].

Variation in size and composition of PGEs result in differences in physical and chemical properties, thus, demonstrating vari- 
ation in electrical properties. Anodization of PGEs in ABS solution leads to the oxidation of the carbon surface [17] and thus limiting current transmitted. CV studies in $\mathrm{K}_{4}\left[\mathrm{Fe}(\mathrm{CN})_{6}\right]$ have indicated that electron transfer rate correlates with an increase in diameter, generating higher amplitude signals used in quantitative determinations, while small diameter PGEs display better reversibility in qualitative investigations.

When the I-V behavior of TRIS-PAGE-PGEs and PAGE-PGEs is compared with the PGEs, a reduction in anodic and cathodic current (Figure 3 ) is demonstrated due to the reduced electron transfer rate of the redoks pair as a result of the polymer coating [16]. The decrease in oxidative and reductive peak current may be due to the changes in load transfer resistance of electrode surface $[19,20]$. In addition, the expected reduction in anodic and cathodic current in the TRIS-PAGE-PGEs has correlated with the incremental increase in TRIS concentration. It must be noted that the I-V behavior of TRIS-PAGE-PGEs in 2 and $2.5 \mathrm{mM}$ TRIS-redox-PBS solution has displayed no apparent change at the higher concentration implying saturation of the polymer surface with TRIS (Figure 2, 3 and 7).

When the impedimetric behavior of TRIS-PAGE-PGEs and PAGE-PGEs is compared with PGEs, a reduction in electron transfer rate of the redox pair was displayed as a result of an increase in impedance (Figure 5) [13]. In addition, the expected increase in the impedance, i.e. the capacitance and diffusion resistance of TRIS-PAGE-PGEs correlated with the incremental increase in TRIS concentration as indicated by the increase in the semicircle diameter and load transfer resistance of the electrode [13]. It must be noted that the decrease in impedance of TRIS-PAGE-PGEs in 2 and 2.5 mM TRIS-redox-PBS solution being lower than expected at the higher concentration implies saturation of the polymer surface with TRIS (Figure 2, 3 and 7).

The CV and EIS results of TRIS-PAGE-PGEs (Figure 2, 3) indicated binding of the chosen analyte (TRIS) to the electrode. In order to determine the specificity of the biosensor to TRIS, the TRIS-PAGE-PGEs were experimented using EDTA. The results of these experiments indicated that the EDTA was not bound to TRIS-PAGE-PGEs since no apparent change in I-V behavior, i.e. a change in anodic and cathodic peak current, correlating with concentration was observed. EDTA, not bound to the electrode surface, remained in solution increasing solution conductivity and subsequently decreasing both solution and thus the total impedance. With the increase in EDTA concentration, the diameter of the semicircular portion of the curve corresponding to solution impedance was reduced and load transfer resistance was decreased.

\section{Conclusions}

In this study, a biosensor consisting of a TRIS-MIP sensor and an electrochemical transducer was developed. Electrode modification and performance was evaluated using CV and EIS. Graphite pencil tip was coated with polyacrylamide gel con- taining TRIS as the analyte and prepared as the working electrode. The electrochemical properties of the electrodes at various concentrations of TRIS and EDTA and $\mathrm{K}_{3}\left[\mathrm{Fe}(\mathrm{CN})_{6}\right]$ redox pair containing phosphate buffer solution, using $\mathrm{Ag} / \mathrm{AgCl}$ reference electrode, platinum counter electrode and working electrode made of graphite pencil tip electrochemical cell were evaluated. The results indicate a correlation between the CV and EIS response and TRIS and EDTA solution concentration. With further research and improvements, the prototypic biosensor selectively specific to TRIS generated in this study can be a promising economical replacement for the commonly used expensive techniques.

\section{Acknowledgements}

We would like to thank Prof.s Dr. Ali Umit Keskin and Mustafa Culha, Assoc. Prof.s Levent Urgan and Erde Can from Yeditepe University, Faculty of Engineering.

\section{References}

1. A. Turner, I. Karube, G. S. Wilson, "Biosensors: Fundamentals and Applications", Oxford University Press, Oxford, U.K., 1987.

2. F. G. Banica, ed., "Chemical Sensors and Biosensors: Fundamentals and Applications", John Wiley \& Sons, Hoboken, New Jersey, U.S.A., 2012. [CrossRef]

3. B. R. Eggins, "Chemical Sensors and Biosensors", John Wiley \& Sons, Hoboken, New Jersey, U.S.A., 2002.

4. S. Yan, Y. Fang, Z. Gao, "Quartz crystal microbalance for the determination of daminozide using molecularly imprinted polymers as recognition element", Biosensors and Bioelectronics, vol. 22, no. 6, pp. 1087-1091, Apr, 2007. [CrossRef]

5. C. Alexander, H. S. Andersson, L. I. Andersson, R. J. Ansell, N. Kirsch, I. A. Nicholls, and M. J. Whitcombe, "Molecular imprinting science and technology: a survey of the literature for the years up to and including 2003", J Molecular Recognition, vol. 19, no. 2, pp. 106-180, Jan, 2006. [CrossRef]

6. W. Li and S. Li, "Oligomers-Polymer Composites-Molecular Imprinting", Springer, Berlin-Heidelberg, Germany, 2006.

7. G. Guan, B. Liu, Z. Wang, Z. Zhang, "Imprinting of molecular recognition sites on nanostructures and its applications in chemosensors", Sensors, vol. 8, no. 12, pp. 8291-8320, Dec, 2008. [CrossRef]

8. L. Ye and K. Haupt, "Molecularly imprinted polymers as antibody and receptor mimics for assays, sensors and drug discovery", Analytical and Bioanalytical Chemistry, vol. 378, no. 8, pp. 1887-1897, Jan, 2004. [CrossRef]

9. A. Bossi, F. Bonini, A. P. F Turner and S. A. Piletsky, "Molecularly imprinted polymers for the recognition of proteins: the state of the art", Biosensors and Bioelectronics, vol. 22, no. 6, pp. 1131-1137, Jan, 2007. [CrossRef]

10. E. Caro, N. Masqué, R. M. Marcé, F. Borrull, P. A. Cormack, and D. C. Sherrington, "Non-covalent and semi-covalent molecularly imprinted polymers for selective on-line solid-phase extraction of 4-nitrophenol from water samples", J Chromatography A, vol. 963, no. 1, pp. 169-178, July, 2002. [CrossRef]

11. G. Vasapollo, R. D. Sole, L. Mergola, M. R. Lazzoi, A. Scardino, S. Scorrano, and G. Mele, "Molecularly imprinted polymers: present and future prospective", Int. J Mol Sci, vol. 12, no. 9, pp. 5908-5945, Sept 14, 2011. [CrossRef] 
12. G. Gomori, "Preparation of Buffers for Use in Enzyme Studies", Methods Enzymol., vol. 1, pp. 138-146, 1955. [CrossRef]

13. E. Barsoukov and J. R. Macdonald, "Impedance spectroscopy: theory, experiment, and applications", John Wiley \& Sons, Hoboken, New Jersey, U.S.A., 2005. [CrossRef]

14. B. Ozcan, B. Demirbakan, G. Yesiller and M.K. Sezginturk, "Introducing a new method for evaluation of the interaction between an antigen and an antibody: Single frequency impedance analysis for biosensing systems", Talanta, vol. 125, pp. 7-13, July, 2014. [CrossRef]

15. S. N. Topkaya, D. Ozkan-Ariksoysal, B. Kosova, R. Ozel and M. Ozsoz, "Electrochemical DNA biosensor for detecting cancer biomarker related to glutathione S-transferase P1 (GSTP1) hypermethylation in real samples", Biosensors and Bioelectronics, vol. 31, no. 1, pp. 516-522, Jan, 2012. [CrossRef]

16. E. Asav and M. K. Sezginturk, "A novel impedimetric disposable immunosensor for rapid detection of a potential cancer bio- marker", Int. J Biological Macromolecules, vol. 66, pp. 273-280, May, 2014. [CrossRef]

17. L. Figueiredo, M. F. R., Pereira, M. M. A. Freitas and J. J. M. Orfao, "Modification of the surface chemistry of activated carbons", Carbon, vol. 37, no. 9, pp. 1379-1389, Dec, 1999. [CrossRef]

18. I. G. David, D.-E. Popa and M. Buleandra, "Pencil Graphite Electrodes: A Versatile Tool in Electroanalysis", J Analytical Methods in Chemistry, https://doi.org/10.1155/2017/1905968, Jan, 2017. [CrossRef]

19. I. S. Park and N. Kim, "Thiolated Salmonella antibody immobilization onto the gold surface of piezoelectric quartz crystal", Biosensors and Bioelectronics, vol. 13, no. 10, pp. 1091-1097, Nov, 1998. [CrossRef]

20. I. Markovich and D. Mandler, "The effect of an alkylsilane monolayer on an indium tin oxide surface on the electrochemistry of hexacyanoferrate", J Electroanalytical Chemistry, vol. 484, no. 2, pp. 194-202, Apr, 2000. [CrossRef]

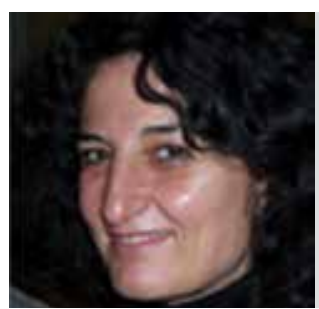

Feride Sermin Utku received her B.S. degree in Biology and Chemistry ID from Earlham College, Richmond, IN, USA, and her MSc and PhD degrees in Biomedical Engineering from Bogazici University, İstanbul, Turkey in 2008. She has been teaching biomaterials at Yeditepe University, İstanbul, Turkey since 2013. Her research interests are biomaterials, surface coatings of implants, hard tissue biomineralization and ceramic materials.

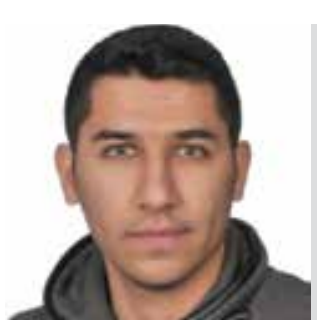

Ozan Enver Özdemir received his B.S. degree in Biomedical Engineering from Yeditepe University, İstanbul, Turkey in 2016. This paper, based on his undergraduate thesis titled "MIP Based Biosensor for Impedance Spectroscopic Analysis" was presented at the Congress of Medical Technologies 2016.

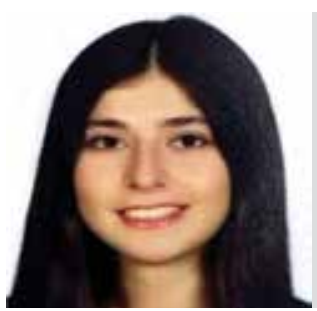

Melahat Sevgül Bakay received her B.S. degree in Biomedical Engineering from Yeditepe University, İstanbul, Turkey in 2016. This paper, based on her undergraduate thesis titled "MIP Based Biosensor for Impedance Spectroscopic Analysis" was presented at the Congress of Medical Technologies 2016. 